\title{
Symmetry-Breaking Plasmonic Metasurfaces for Broadband Light Bending
}

Ni, Xingjie; Emani, Naresh K.; Kildishev, Alexander V.; Boltasseva, Alexandra; Shalaev, Vladimir M.

Published in:

CLEO Technical Digest

Publication date:

2012

Document Version

Publisher's PDF, also known as Version of record

Link back to DTU Orbit

Citation $(A P A)$ :

Ni, X., Emani, N. K., Kildishev, A. V., Boltasseva, A., \& Shalaev, V. M. (2012). Symmetry-Breaking Plasmonic Metasurfaces for Broadband Light Bending. In CLEO Technical Digest (pp. QM3F.1). Optical Society of America.

\section{General rights}

Copyright and moral rights for the publications made accessible in the public portal are retained by the authors and/or other copyright owners and it is a condition of accessing publications that users recognise and abide by the legal requirements associated with these rights.

- Users may download and print one copy of any publication from the public portal for the purpose of private study or research.

- You may not further distribute the material or use it for any profit-making activity or commercial gain

- You may freely distribute the URL identifying the publication in the public portal

If you believe that this document breaches copyright please contact us providing details, and we will remove access to the work immediately and investigate your claim. 


\title{
Symmetry-Breaking Plasmonic Metasurfaces for Broadband Light Bending
}

\author{
Xingjie Ni, ${ }^{1, *}$ Naresh K. Emani, ${ }^{1}$ Alexander V. Kildishev, ${ }^{1}$ Alexandra Boltasseva, ${ }^{1,2}$ and Vladimir M. Shalaev ${ }^{1}$ \\ ${ }^{1}$ School of Electrical and Computer Engineering and Birck Nanotechnology Center, Purdue University, West Lafayette, Indiana 47907, USA \\ ${ }^{2}$ DTU Fotonik, Department of Photonics Engineering, Technical University of Denmark, Lyngby, DK-2800, Denmark \\ xni@purdue.edu
}

\begin{abstract}
We experimentally demonstrate unparalleled wave-front control in a broadband, optical wavelength range from $1.0 \mu \mathrm{m}$ to $1.9 \mu \mathrm{m}$, using a thin plasmonic layer (metasurface) consisting of a nanoantenna array that breaks the symmetry along the interface.

OCIS codes: (160.3918) Metamaterials; (250.5403) Plasmonics; (240.6700) Surfaces
\end{abstract}

\section{Introduction}

The precise manipulation of phase with metasurfaces (thin metamaterial layers) can play a fundamental role in generating synthetic scattering diagrams of macroscopic objects. Thin metasurfaces can introduce additional spatially non-uniform phase in the incoming wave, thereby affecting its scattering characteristics. The amount of phase change is limited by the optical properties of the materials, and significant changes typically require a propagation length comparable to or larger than the wavelength. The nanotechnology revolution in the last decade has made it possible to fabricate metamaterials that are capable of bending light in unusual ways, and hence many fantastic applications are possible such as negative refraction [1], super-resolution lenses [2,3], and cloaking [4]. Still, phase control and light bending by an interface until recently has been dictated by material parameters as governed by the conventional Snell's law. The newly discovered generalized version of Snell's law opens a new era of manipulating light [5]:

$$
\begin{array}{ll}
\text { For refraction: } & \sin \left(\theta_{t}\right) n_{t}-\sin \left(\theta_{i}\right) n_{i}=\lambda \nabla \Phi / 2 \pi, \\
\text { For reflection: } & \sin \left(\theta_{r}\right)-\sin \left(\theta_{i}\right)=n_{i}^{-1} \lambda \nabla \Phi / 2 \pi,
\end{array}
$$

where $\theta_{\mathrm{i}}, \theta_{\mathrm{r}}$, and $\theta_{\mathrm{t}}$ are the incident, reflection, and refraction angles, respectively. $\nabla \Phi$ is a gradient of discontinuity in phase along an interface. This abrupt phase change on the interface can modify the direction of the refracted and the reflected rays at will. In a more general interpretation, $\nabla \Phi$ is essentially an additional momentum contribution that is introduced at the interface by breaking the symmetry; hence, this extra momentum has to be compensated by bending the reflected and refracted light wave in order to conserve momentum. When there is no phase gradient $\nabla \Phi$ along the interface, Eqs. (1) and (2) reproduce the conventional Snell's law. Thus the generalized version of Snell's law indicates that by designing and engineering the phase discontinuity along an interface, one can fully control the bending of light beyond what the conventional Snell's law predicts. Such an interface has been realized at a wavelength of $8 \mu \mathrm{m}$ [5], and we recently also experimentally demonstrated this unparalleled degree of arbitrary wave-front control in a broadband wavelength range from $1.0 \mu \mathrm{m}$ to $1.9 \mu \mathrm{m}$, which is accomplished with a relatively thin 30-nm $(\sim \lambda / 50)$ plasmonic nano-antenna interface. [6]

\section{Experiment and results}

In order to introduce an abrupt phase change on the interface, we use a plasmonic nano-antenna array similar to that in Ref. [5] and consisting of V-shaped gold antennas (Fig. 1). The antisymmetric modes supported by this type of nanoantenna can provide a tunable phase delay for cross-polarized light (whose polarization is perpendicular to the incident polarization) by appropriately choosing the design parameters of the antennas. A unit cell of the nanoantenna array consisting of eight nano-antennas can provide a phase shift from 0 to $2 \pi$.

We fabricated a nano-antenna array sample on a double-side polished silicon substrate with standard electron-beam lithography and lift-off processes. The height of the nano-antennas was about $30 \mathrm{~nm}$ and the arm width was $40 \mathrm{~nm}$. Four large arrays $\left(\sim 500 \times 500 \mu \mathrm{m}^{2}\right)$ with different periodicities were fabricated on the same substrate. The angles of the reflected and refracted light from the sample were measured using the scatterometry mode of a spectroscopic ellipsometer (J. A. Woollam Co., V-VASE). The experimental measurements indicate that the reflection and refraction of the cross-polarized light are both "negative" in a broad range of incidence angles (Figs. 2a and b). All the experimental data matches quite well with the theoretical predictions given by the generalized Snell's law [5]. 

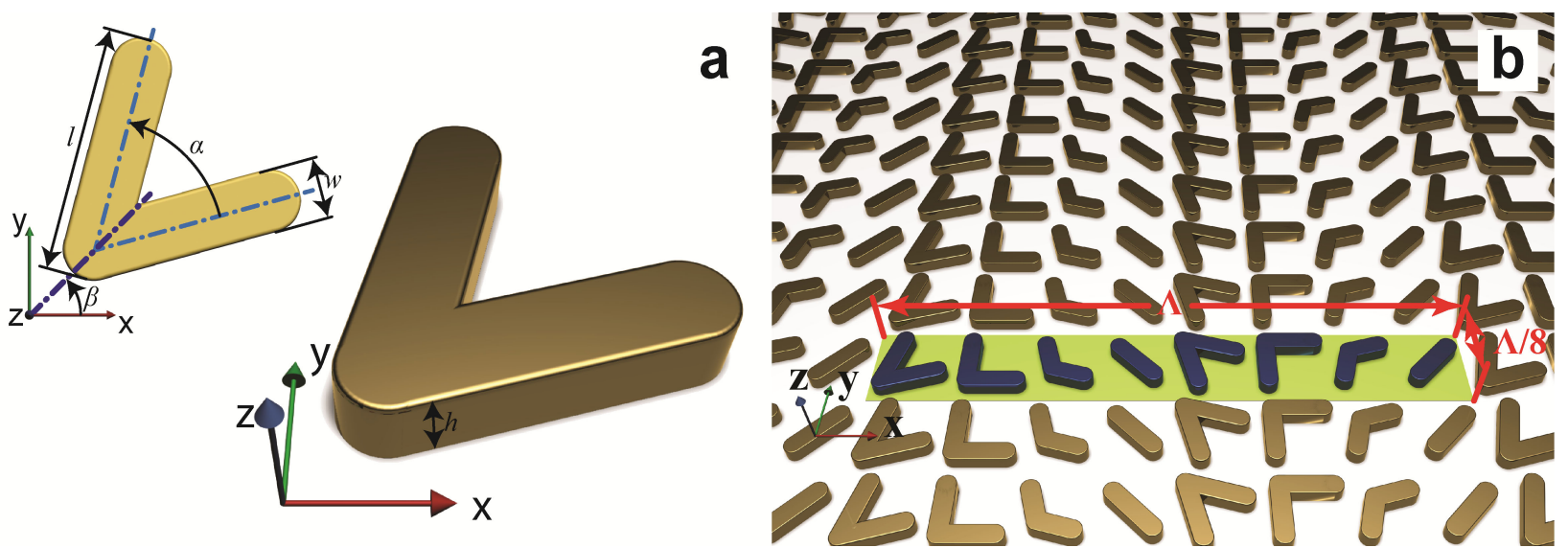

Fig. 1. (a) Schematic view of a constituent antenna, which has two rectangular arms split with an angle $\alpha$ forming a V shape. (b) Schematic view of a representative antenna array. The unit cell of the plasmonic interface (in blue) consists of eight gold $\mathrm{V}$-antennas of 40-nm arm width and 30$\mathrm{nm}$ thickness, repeated with a periodicity of $\Lambda$ in the $\mathrm{x}$ direction and $\Lambda / 8$ in the $\mathrm{y}$ direction. In a unit cell, the first four antennas have $\beta=45^{\circ}, \alpha=$ $60^{\circ}, 90^{\circ}, 120^{\circ}, 180^{\circ}$. The latter four antennas in the unit cell have $\beta=-45^{\circ}$, and the rest of the parameters are the same as those of the first four antennas. The phase delay for cross-polarized light increases along the $\mathrm{x}$ axis from right to left.
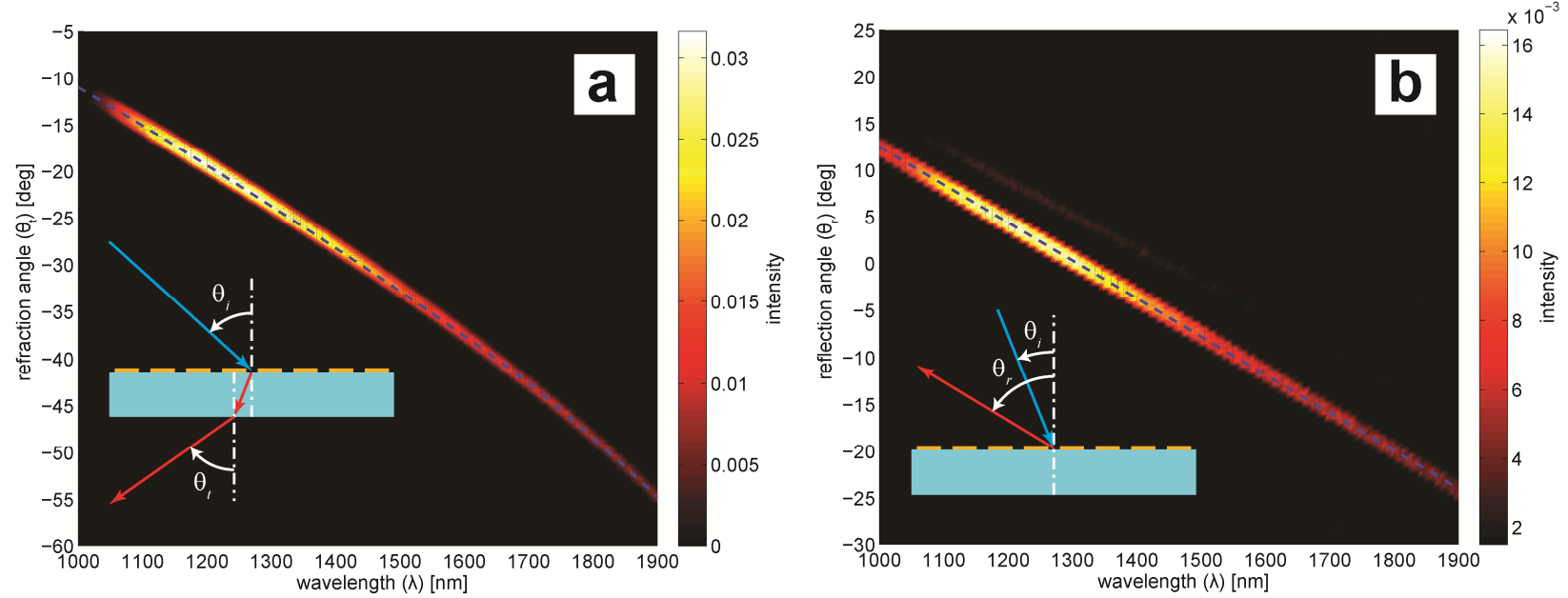

Fig. 2. False-color maps indicating the experimentally measured relative intensity profiles for the antenna array with $\Lambda=1440 \mathrm{~nm}$ and $\mathrm{x}$ polarized excitation. The dashed line shows the theoretical prediction of the peak position using the generalized Snell's law. (a) Refraction angle versus wavelength for cross-polarized light with $30^{\circ}$ incidence angle. (b) Reflection angle versus wavelength for cross-polarized light with $65^{\circ}$ incidence angle.

\section{Conclusion}

We demonstrated that by providing a phase shift using a symmetry-breaking metasurface consisting of a plasmonic nano-antenna array, the optical wave front can be modified in an arbitrary way. Thus, unparalleled control of anomalous reflection and refraction, including negative refraction, is possible using such metasurfaces. The experimental results show that our design operates in a broad range of wavelengths from $1.0 \mu \mathrm{m}$ to $1.9 \mu \mathrm{m}$. We truly believe this technique could have an important impact on nano-photonic and optical-metamaterial applications.

\section{Acknowledgment}

This work was supported by AFOSR-MURI (FA9550-10-1-0264) and NSF-DMR (1120923).

[1] V. G. Veselago, "The Electrodynamincs of Substances with Simultaneously Negative Values of $\varepsilon$ and $\mu$," Soviet Physics Uspekhi 10, 509 (1968).

[2] J. B. Pendry, "Negative Refraction Makes a Perfect Lens," Physical Review Letters 85, 3966 (2000).

[3] X. Zhang, Z. W. Liu, H. Lee, Y. Xiong, and C. Sun, "Far-field optical hyperlens magnifying sub-diffraction-limited objects," Science 315, 1686-1686 (2007).

[4] J. B. Pendry, D. Schurig, and D. R. Smith, "Controlling Electromagnetic Fields," Science 312, 1780-1782 (2006).

[5] N. Yu, P. Genevet, M. A. Kats, F. Aieta, J.-P. Tetienne, F. Capasso, and Z. Gaburro, "Light Propagation with Phase Discontinuities: Generalized Laws of Reflection and Refraction," Science 334, 333-337 (2011).

[6] X. Ni, N. K. Emani, A. V. Kildishev, A. Boltasseva, and V. M. Shalaev, "Broadband Light-Bending with Plasmonic Nanoantennas," (to be published). 